\title{
Potential predictors for health-related quality of life in stroke patients undergoing inpatient rehabilitation
}

\author{
Chien-Min Chen ${ }^{1,2}$, Chih-Chien Tsai ${ }^{3,4}$, Chia-Ying Chung ${ }^{2,3}$, Chia-Ling Chen ${ }^{3,4^{*}}$, Katie PH Wu W $^{2,3}$ \\ and Hsieh-Ching Chen ${ }^{5}$
}

\begin{abstract}
Background: Improving HRQOL is the desired outcome for patients with stroke undergoing inpatient rehabilitation services. This study aimed to comprehensively identify the potential health-related quality of life (HRQOL) predictors in patients with stroke undergoing inpatient rehabilitation within the first year after stroke; thus far, such an investigation has not been conducted.
\end{abstract}

Methods: We enrolled 119 patients (88 males, 31 females) with stroke, and examined 12 potential predictors: age, sex, stroke type, stroke side, duration after onset, cognition (Mini-Mental State Examination; MMSE), depression (Beck Depression Inventory-II), stroke severity (National Institutes of Health Stroke Scale; NIHSS), upper- and lower-extremity motor function scores of the Fugl-Meyer Assessment (FMA) scale, balance (Berg Balance Scale; BBS), and functional status (Functional Independence Measure). HRQOL was measured using Stroke Impact Scale (SIS) 3.0.

Results: NIHSS score predicted the strength domain and total SIS score $(41.5 \%$ and $41.7 \%$ of the variances, respectively). BBS score was a major predictor of mobility and participation/role domains (48.6\% and $10 \%$ of the variances, respectively). MMSE score predicted the memory and communication domains ( $22.5 \%$ and $36.3 \%$ of the variances, respectively). Upper extremity score of the FMA scale predicted the daily living/instrumental activities of daily life and hand function domains (40.3\% and $20.6 \%$ of the variances, respectively). Stroke side predicted the emotion domain (11.6\% of the variance).

Conclusions: NIHSS, MMSE, BBS, FMA, and stroke side predicted most HRQOL domains. These findings suggest that different factors predicted various HRQOL domains in patients with stroke.

Keywords: Stroke, Predictor, Health-Related Quality of Life, Rehabilitation

\section{Background}

Stroke is a major health problem across the world. It has been long established that stroke has wide-ranging effects in physical, mental, and social life domains [1]. Healthrelated quality of life (HRQOL) refers to the health impacts on an individual's functioning and on his or her perceived wellness in various life domains [2]. Stroke

\footnotetext{
* Correspondence: clingchen@gmail.com

${ }^{3}$ Department of Physical Medicine and Rehabilitation, Chang Gung Memorial Hospital, Linkou, No.5, Fushing St. Kuei-Shan Dist., Taoyuan City 33305, Taiwan ${ }^{4}$ Graduate Institute of Early Intervention, College of Medicine, Chang Gung University, Taoyuan, No.259, Wunhua 1st Rd. Kuei-Shan Dist., Taoyuan City 33302, Taiwan

Full list of author information is available at the end of the article
}

survivors have lower mean scores for physical health $(-7.9 \%)$, mental health (-4.1\%), and health utility (-6.9\%) of HRQOL than the nonstroke population [3].

According to the review of literature, age [4], sex [4], stroke type [5], stroke side [6], duration after onset [7], stroke severity [8], functional status [9], upper extremity (UE) motor function [10], balance function [11], lower extremity (LE) motor function [12], cognition function [8], and depression [13] have been identified as potential predictors of HRQOL outcomes in patients with stroke. The potential HRQOL predictors are varied among studies possibly because of different predictors and HRQOL domains investigated in each study. For example, 
age was identified as an HRQOL predictor for patients with stroke in Strum et al.'s study [4] but not in Fatoye et al.'s study [14]. To the best of our knowledge, only few studies $[15,16]$ have investigated a comprehensive list of HRQOL predictors in various domains in patients with stroke.

Recovery in stroke patients receiving rehabilitation primarily occurs in the first 3 months after stroke and continues in the following 3 months [17]. In addition, $6.5 \%$ patients with stroke surviving for at least 1 year after stroke have been reported to show improvements in the 6-12 months after stroke [17]. Improving HRQOL is also the desired outcome for patients with stroke undergoing inpatient rehabilitation services. Mackenzie et al. [18] suggested that HRQOL predictors in patients with stroke admitted to rehabilitation hospitals during 0.5-3 months following stroke were the functional ability, physical, and psychological dimensions of HRQOL. Froes et al. [13] evaluated the factors affecting HRQOL [using Short Form-36 (SF-36)] in 64 patients ( $\geq 6$ months after stroke) admitted to a rehabilitation program. They found that functional status affected physical functioning and physical role, and that depression could affect vitality, social function, emotional role, and mental health [13]. HRQOL after stroke varies considerably between different countries $[19,20]$. This may be due to differences in the guidelines and strategies for post-stroke care and rehabilitation services. Compliance to post-acute-stroke rehabilitation guidelines could also be a quality-of-care indicator and affect HRQOL in patients with stroke undergoing inpatient rehabilitation [21]. Hopman et al. [22] reported that all 8 domains of the SF-36 improved in patients with stroke during inpatient rehabilitation; however, five domains declined at 6 months follow-up after discharge. Post-acute-stroke rehabilitation plays an important role in HRQOL. In Taiwan, the health care policy (National Health Insurance) allows most poststroke patients to be admitted for inpatient rehabilitation several times within first year after a stroke. However, articles have comprehensively investigated HRQOL predictors for patients with stroke who were currently undergoing inpatient stroke rehabilitation during the first year after stroke are still lacking.

Many scales, including generic scales, such as SF-36 and EuroQol-5 Dimension Questionnaire (EQ-5D) [23-25], and stroke-specific [26] scales, have been used to assess HRQOL in patients with stroke. SF-36 was the most widely used generic HRQOL measurement [25]. However, the ceiling and floor effects that exist in some SF-36 domains [27] limit its use in the evaluation of patients with stroke. The Stroke Impact Scale (SIS) 3.0 is a comprehensive stroke-specific HRQOL measurement instrument [28]. SIS was developed to assess eight domains for mild-tomoderate stroke patients and it has been used in Taiwan $[29,30]$.

Identifying potential HRQOL predictors in patients with stroke will allow clinicians to predict HRQOL outcomes and plan early and appropriate treatment strategies. The research question was whether potential predictors for HRQOL differed among various domains in patients with stroke undergoing inpatient rehabilitation. After reviewing past articles, 12 potential predictors were selected for examination: age (at assessment), sex, stroke type (hemorrhagic or ischemic), stroke side (left or right), duration after onset, stroke severity, cognition function, depression, balance, UE and LE motor function, and functional status. We measured HRQOL with the SIS 3.0. We hypothesized that different predictors are associated with various HRQOL domains in patients undergoing inpatient stroke rehabilitation during the 1st year after stroke. The aim of this study was to reveal a comprehensive list of determinant factors for various HRQOL domains and total HRQOL score for patients receiving inpatient rehabilitation within 1 year after stroke.

\section{Methods}

\section{Participants}

Participants with stroke were recruited from the inpatient rehabilitation ward of a tertiary hospital. The diagnosis of stroke, lesion side, and stroke type were confirmed by a physiatrist by history evaluation, physical examination, brain imaging, and chart review. The physiatrist also determined a patient's eligibility for the study. The inclusion criteria were (a) first-time unilateral cerebral stroke, (b) stroke onset $\leq 1$ year, (c) admission to the rehabilitation ward, (d) ages 30-80 years, and (e) no active medical problems or physical conditions. The exclusion criteria were (a) brainstem or cerebellar stroke, (b) severe cognitive impairment, (c) severe aphasia, and (d) poor cooperation with assessments. Patients with brainstem or cerebellar stroke were excluded from the study because the symptoms differ from the typical hemiplegia or hemiparesis of cerebral stroke. Of the 258 patients with stroke initially assessed for eligibility, 121 were excluded because they did not meet the criteria and 18 declined to participate in the study. In total, 119 patients with stroke (88 males, 31 females) were included. The study protocol was approved by the Institutional Review Board for Human Studies at Chang Gung Memorial Hospital. All participants signed informed consent forms before enrollment.

\section{Assessment procedures}

Two trained raters (a physical therapist and an occupational therapist) administered all measures. Raters reviewed 
the written instructions and were trained through repeated practice; a senior certified physical therapist or a senior certified occupational therapist assessed rater competence. All potential predictors were identified and HRQOL outcomes were measured for each study participant 2 to 3 weeks after admission for inpatient rehabilitation. All measurements for each participant were completed on the same day. The rehabilitation program offered services including both physical and occupational therapies. In addition, speech therapy was provided if required. Each of the daily physical, occupational, and speech therapy sessions usually lasted over 50 minutes on weekdays. Physical therapy included facilitation techniques, balance and transfer training, therapeutic exercise, strengthening exercise, and ambulation training. Occupational therapy involved motor task training, coordination training, hand function training, daily activities training, and visual perception training. Speech therapy consisted of auditory comprehension training, cognitive training, verbal production, oromotor coordination, augmentative communication, swallowing, and feeding training.

\section{Outcome measure of HRQOL}

The SIS 3.0 was used for HRQOL measurement. SIS is a stroke-specific and self-reported instrument [31], consisting of 59 items designed for assessing 8 functional domains, including strength, memory, emotion, communication, activities of daily living (ADL)/instrumental activities of daily living (IADL), mobility, hand function, and participation/ role. The internal consistency (Cronbach $\alpha: 0.94)$ and validity of SIS 3.0 was proved to be adequate. Participants responded to items in each domain using a 5-point rating scale. For strength items, 1 point meant "no strength at all" and 5 points meant "a lot of strength." For memory, communication, ADL/IADL, mobility, and hand function items, 1 point meant "extremely difficult/cannot do at all" and 5 points meant "not difficult at all." For emotion and participation/role items, 1 point meant "all of the time" and 5 points meant "none of the time." Each domain of SIS has a range of $0-100$ with higher scores indicated better QOL. Twenty-eight items in the four domains of strength, hand function, ADL/IADL, and mobility were combined to produce the composite physical domain.

\section{Potential predictors}

The Mini-Mental State Examination (MMSE) [32] was used to measure cognition function, the Beck Depression Inventory (BDI)-II [33] was used to measure depression status, the National Institutes of Health Stroke Scale (NIHSS) [34] was used to measure stroke severity, the Berg Balance Scale (BBS) [35] was used to measure balance function, the Fugl-Meyer Assessment (FMA) [36]-UE motor function was used to measure UE motor function, the FMA-LE motor function was used to measure LE motor function, and the Functional Independence Measure (FIM) [37] was used to measure functional status.

\section{MMSE}

MMSE [32] is a brief 11-question test widely used for the evaluation of cognitive impairment. It measures orientation, registration, attention and calculation, recall, and language. The maximum total score is 30 points with lower total scores suggesting more severe cognitive impairment.

\section{BDI-II}

BDI-II [33] is a 21-item inventory that assesses the severity of depressive symptoms. Each item is rated on a 4-point scale range of 0-3. A score of 0 means "not feeling sadness at all" and 3 means "intolerable sadness or unhappiness." The total score range on the BDI-II is $0-63$.

\section{NIHSS}

NIHSS [34] is a 15-item assessment tool that provides a quantitative measure of stroke-related neurological deficit. NIHSS evaluates levels of consciousness, visual field loss, extraocular movement, facial muscle function, UE and LE motor strength, coordination, aphasia, dysarthria, sensory loss, extinction, and loss of attention. Total NIHSS scores range from 0 to 42, with higher scores meaning more severe neurological deficit.

\section{BBS}

BBS [35] is a 14-item test for evaluating static and dynamic balance ability. Score range of each item is 0-4; a score of 0 meant "unable to perform the task" and a score of 4 meant "able to independently perform the task without difficulty." The final measure is the sum of all scores, with the lowest and highest possible total scores being 0 and 56 , respectively.

\section{FMA}

FMA [36] is a stroke-specific, performance-based impairment index. It is designed to assess motor functioning, balance, sensation, and joint functioning in patients with post-stroke hemiplegia. Scoring is based on direct observation of performance. Scale items are scored on the basis of ability to complete the task using a 3-point scale where $0=$ cannot perform, $1=$ performs partially, and $2=$ performs fully. FMA has a score range of $0-100$ (66 UE, $34 \mathrm{LE}$ ), with 100 indicating normal motor performance.

\section{FIM}

The FIM [37] is an instrument designed for functional status and frequently used for patients with stroke. The FIM includes 18 items, each with a maximum score of 7 
and a minimal score of 1 . A score of 7 means complete independence and a score of 1 means complete dependence. Possible FIM total scores range from 18 to 126. Higher score means more independent functional status.

\section{Statistical analysis}

Statistical analyses were performed with SPSS 18.0 for Windows. The dependent variables were the SIS 3.0. A two-step process determined whether a variable was considered a predictor of outcome measurements. First, a Pearson correlation coefficient $(r)$ determined correlations between potential predictors and scores on the outcome measures (8 domains and total score). A $P$ value of 0.05 was required for inclusion in the regression analysis. Potential predictors were used in a stepwise procedure to generate a linear regression model for outcome measurements. Adjusted $R^{2}, P$ values, and regression coefficients $(\beta)$ were used to assess goodness-of-fit in the regression models. Regression diagnostics were also performed to check the multicollinearity among the predictors in the regression models.

\section{Results}

Table 1 lists the demographic and clinical characteristics of the patients (mean age 54.89 years, standard deviation: 10.47 years).

\section{Correlation between potential predictors and HRQOL}

Table 2 lists Pearson correlation coefficients of the 12 possible predictors and the scores on various QOL outcome measures. Age and sex were not associated with any domain of SIS. Stroke type was associated with communication and hand function (patients with infarction had better communication and hand function than patients with hemorrhage). Stroke side was associated with emotion, mobility, participation/role, and total SIS score (patients with left side lesion had better scores in these domains than those with right side lesion). Duration after onset was associated with strength (longer duration after onset leads to less strength). All clinical predictors were associated with most domains of SIS.

\section{Potential predictors of predicting HRQOL by regression analysis}

Table 3 presents the results of the stepwise multiple regression analyses. NIHSS score, FIM score, and duration after onset were predictors in the strength domain of the SIS (adjusted $\left.R^{2}=0.540\right)(P<0.001)$. MMSE and BDI-II scores were predictors in the memory domain of the SIS (adjusted $\left.R^{2}=0.290\right)(P<0.001)$. Stroke side and BDI-II score were predictors in the emotion domain of the SIS (adjusted $\left.R^{2}=0.169\right) \quad(P<0.001)$. MMSE score, stroke type, and FMA-UE score were predictors in the communication domain of the SIS (adjusted $R^{2}=0.413$ )
Table 1 Demographic characteristics, clinical characteristics, and health-related quality of life in patients with stroke

\begin{tabular}{|c|c|}
\hline & Value $^{a}$ \\
\hline & Mean (SD) or number (\%) \\
\hline \multicolumn{2}{|l|}{ Demographic characteristics } \\
\hline Age (years) & $54.89(10.47)$ \\
\hline \multicolumn{2}{|l|}{ Sex } \\
\hline Male & $88(73.9 \%)$ \\
\hline Female & 31 (26.1\%) \\
\hline \multicolumn{2}{|l|}{ Clinical characteristics } \\
\hline \multicolumn{2}{|l|}{ Stroke type } \\
\hline Hemorrhage & $62(52.1 \%)$ \\
\hline Infarction & $57(47.9 \%)$ \\
\hline \multicolumn{2}{|l|}{ Stroke side } \\
\hline Left & $59(49.6 \%)$ \\
\hline Right & $60(50.4 \%)$ \\
\hline Duration after onset (months) & $6.04(3.24)$ \\
\hline MMSE & $26.39(4.17)$ \\
\hline BDI-II & $14.65(9.81)$ \\
\hline $\mathrm{NIHSS}$ & $4.31(3.00)$ \\
\hline BBS & 38.45 (14.99) \\
\hline FMA-UE & $35.27(21.86)$ \\
\hline FMA-LE & $31.59(10.00)$ \\
\hline FIM & $88.25(26.29)$ \\
\hline \multicolumn{2}{|l|}{ SIS } \\
\hline Strength & $8.71(3.01)$ \\
\hline Memory & $27.71(6.03)$ \\
\hline Emotion & $28.54(5.43)$ \\
\hline Communication & $30.37(5.04)$ \\
\hline $\mathrm{ADL} / \mathrm{IADL}$ & $29.26(8.84)$ \\
\hline Mobility & $28.72(9.74)$ \\
\hline Hand function & $9.92(6.03)$ \\
\hline Participation/Role & $22.49(9.02)$ \\
\hline Total & $185.72(34.14)$ \\
\hline
\end{tabular}

${ }^{\mathrm{a}}$ Continuous and categorical variables were displayed as mean (SD) and number (\%), respectively

MMSE Mini-Mental State Examination; BDI-II Beck Depression Inventory-II; NIHSS National Institute of Health Stroke Scale; BBS Berg Balance Scale; FMA Fugl-Meyer Assessment; UE upper extremity; LE lower extremity; FIM Functional Independence Measure; SIS Stroke Impact Scale; $A D L$ activities of daily living; $I A D L$ instrumental activities of daily living; $S D$ standard deviation

$(P<0.001)$. FMA-UE, FIM, and BBS scores were positive predictors in the ADL/IADL domain of the SIS (adjusted $\left.R^{2}=0.532\right)(P<0.001)$. BBS, FIM, FMA-LE, and BDI-II scores were predictors in the mobility domain of the SIS (adjusted $\left.R^{2}=0.605\right) \quad(P<0.001)$. FMA-UE and FIM scores were positive predictors of hand function in the SIS (adjusted $\left.R^{2}=0.240\right)(P<0.001)$. BBS score was a positive predictor in the participation/role domain of the 
Table 2 Relationships between the potential predictors and SIS domains in patients with stroke

\begin{tabular}{|c|c|c|c|c|c|c|c|c|c|}
\hline \multirow[t]{3}{*}{ Candidate predictors } & \multicolumn{9}{|c|}{ Pearson's $r$} \\
\hline & \multicolumn{9}{|c|}{ SIS domains } \\
\hline & Strength & Memory & Emotion & Communication & ADL/IADL & Mobility & Hand function & Participation/Role & Total \\
\hline \multicolumn{10}{|l|}{ Demographic } \\
\hline Age & 0.006 & 0.096 & -0.099 & 0.123 & -0.033 & -0.135 & 0.082 & 0.067 & 0.005 \\
\hline Sex & 0.056 & -0.071 & -0.052 & -0.017 & 0.028 & -0.036 & 0.053 & -0.030 & -0.020 \\
\hline Stroke type & 0.002 & 0.079 & 0.023 & $0.218^{*}$ & 0.063 & 0.092 & $0.221^{*}$ & 0.043 & 0.143 \\
\hline Stroke side & -0.134 & -0.084 & $-0.352^{*}$ & 0.056 & -0.133 & $-0.188^{*}$ & -0.025 & $-0.257^{*}$ & $-0.234^{*}$ \\
\hline Duration after onset & $-0.215^{*}$ & -0.163 & -0.066 & -0.152 & 0.028 & 0.051 & -0.009 & 0.012 & -0.057 \\
\hline \multicolumn{10}{|l|}{ Clinical } \\
\hline MMSE & $0.423^{*}$ & $0.481^{*}$ & 0.109 & $0.607^{*}$ & $0.433^{*}$ & $0.470^{*}$ & $0.272^{*}$ & 0.124 & $0.556^{*}$ \\
\hline BDI-II & -0.158 & $-0.298^{*}$ & $-0.293^{*}$ & $-0.202^{*}$ & $-0.200^{*}$ & $-0.234^{*}$ & -0.086 & -0.095 & $-0.302^{*}$ \\
\hline NIHSS & $-0.648^{*}$ & $-0.332^{*}$ & $-0.196^{*}$ & $-0.425^{*}$ & $-0.570^{*}$ & $-0.540^{*}$ & $-0.382^{*}$ & $-0.267^{*}$ & $-0.649^{*}$ \\
\hline BBS & $0.544^{*}$ & $0.192^{*}$ & $0.214^{*}$ & $0.245^{*}$ & $0.600^{*}$ & $0.701^{*}$ & $0.312^{*}$ & $0.328^{*}$ & $0.649^{*}$ \\
\hline FMA-UE & $0.599^{*}$ & $0.338^{*}$ & 0.109 & $0.416^{*}$ & $0.639^{*}$ & $0.554^{*}$ & $0.461^{*}$ & $0.189^{*}$ & $0.646^{*}$ \\
\hline FMA-LE & $0.536^{*}$ & $0.371^{*}$ & 0.109 & $0.447^{*}$ & $0.584^{*}$ & $0.633^{*}$ & $0.387^{*}$ & 0.167 & $0.640^{*}$ \\
\hline FIM & $0.507^{*}$ & $0.363^{*}$ & 0.165 & $0.386^{*}$ & $0.580^{*}$ & $0.600^{*}$ & $0.403^{*}$ & $0.235^{*}$ & $0.647^{*}$ \\
\hline
\end{tabular}

${ }^{*} P$-values $<0.05$

MMSE Mini-Mental State Examination; BDI-II Beck Depression Inventory-II; NIHSS National Institute of Health Stroke Scale; BBS Berg Balance Scale; FMA Fugl-Meyer Assessment; UE upper extremity; $L E$ lower extremity; FIM Functional Independence Measure; SIS Stroke Impact Scale; $A D L$ activities of daily living; IADL instrumental activities of daily living

SIS (adjusted $\left.R^{2}=0.100\right)(P<0.001)$. NIHSS, FIM, FMALE, BDI-II, MMSE, and BBS scores were predictors of the total SIS score (adjusted $\left.R^{2}=0.677\right)(P<0.001)$.

The nine final regression equations were as follows:

$$
\begin{aligned}
\text { Strength }= & 8.741-(0.460) \text { NIHSS } \\
& +(0.038) \text { FIM }-(0.212) \text { duration after onset }
\end{aligned}
$$

$$
\begin{aligned}
& \text { Memory }=13.927+(0.626) \text { MMSE- }-(0.157) \text { BDI-II } \\
& \text { Emotion }=35.711-(3.412) \text { stroke side }
\end{aligned}
$$$$
\text { (left : } 1 \text {; right : } 2)-(0.130) \text { BDI-II }
$$

Communication $=11.233+(0.598)$ MMSE

$$
+(1.162) \text { stroke type }
$$

(hemorrhage : 1 ; infarction : 2)

$$
+(0.039) \text { FMA-UE }
$$

$$
\begin{aligned}
& \mathrm{ADL} / \mathrm{IADL}=11.335+(0.146) \mathrm{FMA}-\mathrm{UE} \\
& +(0.088) \mathrm{FIM}+(0.147) \mathrm{BBS} \\
& \text { Mobility }=7.809+(0.262) \text { BBS }+(0.088) \text { FIM } \\
& +(0.185) \text { FMA-LE-(0.123) BDI-II } \\
& +(0.053) \text { FIM }
\end{aligned}
$$

$$
\begin{aligned}
\text { Total }= & 112.671-(2.434) \text { NIHSS }+(0.392) \text { FIM } \\
& +(0.402) \text { FMA-LE }-(0.555) \text { BDI-II } \\
& +(1.310) \text { MMSE }+(0.352) \text { BBS }
\end{aligned}
$$

\section{Discussion}

This is the first study on the potential predictors of various HRQOL domains in patients with stroke receiving inpatient rehabilitation during the first year after stroke. BBS score was a major factor in predicting the mobility and participation/role. FMA-UE scores were a major factor in predicting hand function and ADL/IADL domains, respectively. MMSE score was the main factor in predicting the memory and communication domains. NIHSS score was a major factor in predicting the strength domain. Stroke side was a major factor in predicting the emotion domain. Our findings suggested that different factors predicted the various HRQOL domains in patients with stroke. The BBS, FMA-UE, MMSE, NIHSS scores, and stroke side predicted most HRQOL domains. This information may allow clinicians to plan specific treatment strategies for patients with stroke.

Our findings are partially in agreement with previous studies [13, 16, 18]. Using SIS as the HRQOL measure, Carod-Artal et al. [16] surveyed the predictors for HRQOL in patients with stroke at outpatient neurology and stroke rehabilitation clinics. They found that the NIHSS and functional status (Barthel Index) were the main predictors for 
Table 3 The predictors for the health-related quality of life in patients with stroke by stepwise multiple regression analyses

\begin{tabular}{|c|c|c|c|c|c|c|c|c|}
\hline $\begin{array}{l}\text { Dependent } \\
\text { variables }\end{array}$ & $\begin{array}{l}\text { Independent } \\
\text { variables }\end{array}$ & $\begin{array}{l}\text { Unstandardized } \\
\text { coefficient ( } \beta \text { ) }\end{array}$ & $\begin{array}{l}\text { standardized } \\
\text { coefficient ( } \beta \text { ) }\end{array}$ & $\begin{array}{l}\text { Lower limit of } \\
95 \% \mathrm{Cl}\end{array}$ & $\begin{array}{l}\text { Upper limit of } \\
95 \% \mathrm{Cl}\end{array}$ & $\begin{array}{l}\text { Adjusted } \\
R^{2}\end{array}$ & $F$ & $P$ \\
\hline \multicolumn{9}{|l|}{ SIS domains } \\
\hline \multirow[t]{5}{*}{ Strength } & & & & & & & 47.094 & $<0.001$ \\
\hline & Constant & 8.741 & & 6.983 & 10.500 & & & \\
\hline & NIHSS & -0.460 & -0.514 & -0.580 & -0.340 & 0.415 & & \\
\hline & FIM & 0.038 & 0.333 & 0.023 & 0.054 & 0.491 & & \\
\hline & $\begin{array}{l}\text { Duration after } \\
\text { onset }\end{array}$ & -0.212 & -0.228 & -0.328 & -0.096 & 0.540 & & \\
\hline \multirow[t]{4}{*}{ Memory } & & & & & & & 25.154 & $<0.001$ \\
\hline & Constant & 13.927 & & 8.187 & 19.668 & & & \\
\hline & MMSE & 0.626 & 0.464 & 0.418 & 0.834 & 0.225 & & \\
\hline & BDI-II & -0.157 & -0.267 & -0.247 & -0.066 & 0.290 & & \\
\hline \multirow[t]{4}{*}{ Emotion } & & & & & & & 12.986 & $<0.001$ \\
\hline & Constant & 35.711 & & 32.692 & 38.729 & & & \\
\hline & Stroke side & -3.412 & -0.315 & -0.292 & -5.232 & 0.116 & & \\
\hline & BDI-II & -0.130 & -0.246 & -0.292 & -0.219 & 0.169 & & \\
\hline \multirow[t]{5}{*}{ Communication } & & & & & & & 28.709 & $<0.001$ \\
\hline & Constant & 11.233 & & 6.551 & 15.914 & & & \\
\hline & MMSE & 0.598 & 0.530 & 0.426 & 0.771 & 0.363 & & \\
\hline & Stroke type & 1.612 & 0.160 & 0.184 & 3.040 & 0.394 & & \\
\hline & FMA-UE & 0.039 & 0.173 & 0.004 & 0.075 & 0.413 & & \\
\hline \multirow[t]{5}{*}{ ADL/IADL } & & & & & & & 45.772 & $<0.001$ \\
\hline & Constant & 11.335 & & 7.333 & 15.338 & & & \\
\hline & FMA-UE & 0.146 & 0.365 & 0.084 & 0.208 & 0.403 & & \\
\hline & FIM & 0.088 & 0.261 & 0.036 & 0.139 & 0.492 & & \\
\hline & BBS & 0.147 & 0.266 & 0.059 & 0.234 & 0.532 & & \\
\hline \multirow[t]{6}{*}{ Mobility } & & & & & & & 46.223 & $<0.001$ \\
\hline & Constant & 7.809 & & 3.172 & 12.446 & & & \\
\hline & BBS & 0.262 & 0.431 & 0.171 & 0.354 & 0.486 & & \\
\hline & FIM & 0.088 & 0.238 & 0.036 & 0.141 & 0.560 & & \\
\hline & FMA-LE & 0.185 & 0.227 & 0.061 & 0.310 & 0.592 & & \\
\hline & BDI-II & -0.123 & -0.130 & -0.234 & -0.013 & 0.605 & & \\
\hline \multirow[t]{4}{*}{ Hand function } & & & & & & & 19.644 & $<0.001$ \\
\hline & Constant & 2.269 & & -1.095 & 5.634 & & & \\
\hline & FMA-UE & 0.094 & 0.347 & 0.045 & 0.144 & 0.206 & & \\
\hline & FIM & 0.053 & 0.231 & 0.011 & 0.095 & 0.240 & & \\
\hline \multirow{3}{*}{$\begin{array}{l}\text { Participation/ } \\
\text { Role }\end{array}$} & & & & & & & 14.152 & $<0.001$ \\
\hline & Constant & 15.420 & & 11.388 & 19.452 & & & \\
\hline & BBS & 0.185 & 0.328 & 0.088 & 0.283 & 0.100 & & \\
\hline \multirow[t]{4}{*}{ Total } & & & & & & & 42.236 & $<0.001$ \\
\hline & Constant & 112.671 & & 83.405 & 141.937 & & & \\
\hline & NIHSS & -2.434 & -0.240 & -3.930 & -0.938 & 0.417 & & \\
\hline & FIM & 0.392 & 0.302 & 0.223 & 0.561 & 0.602 & & \\
\hline
\end{tabular}


Table 3 The predictors for the health-related quality of life in patients with stroke by stepwise multiple regression analyses (Continued)

$\begin{array}{llllll}\text { FMA-LE } & 0.402 & 0.140 & -0.013 & 0.817 & 0.633 \\ \text { BDI-II } & -0.555 & -0.167 & -0.911 & -0.199 & 0.650 \\ \text { MMSE } & 1.310 & 0.171 & 0.346 & 2.274 & 0.668 \\ \text { BBS } & 0.352 & 0.165 & 0.017 & 0.686 & 0.677\end{array}$

MMSE Mini-Mental State Examination; BDI-II Beck Depression Inventory-II; NIHSS National Institute of Health Stroke Scale; FMA Fugl-Meyer Assessment; UE upper extremity; $L E$ lower extremity; $B B S$ Berg Balance Scale; FIM Functional Independence Measure; SIS Stroke Impact Scale; $A D L$ activities of daily living; IADL instrumental activities of daily living; $C l$ confidence interval

the strength domain, the MMSE and the hospital anxiety and depression scales were the main predictors for the memory domain, the hospital anxiety and depression scale were the main predictors for the emotional domain, MMSE was a predictor for the communication domain, functional status (Barthel Index) and the hospital anxiety and depression scale were predictors for the mobility domain, and functional status (Barthel Index) was a predictor for the ADL/IADL and hand function domains. Froes et al. [13] and Mackenzie et al. [18] surveyed predictors for HRQOL in patients with stroke currently undergoing inpatient rehabilitation. Froes et al. [13] showed that FIM and time after stroke onset could predict the physical functioning domain, and that the BDI-II was a major predictor for vitality, emotional role, and mental health domains of SF-36. Using the sickness impact profile (SIP) as the HRQOL measure, Mackenzie et al. found that baseline and 2-week functional status (Modified Barthel Index) could be a predictor for total SIP score at 2 weeks and 3 months individually after admission [18]. Some inconsistent results among studies may be due to the patient characteristics (stroke types and lesion sides), rehabilitation programs (e.g. inpatient or ambulatory rehabilitation), onset time after stroke, selected HRQOL assessment tools, selected predictors, etc.

In this study, BBS predicted variances in several domains of HRQOL, such as participation/role and mobility domains, explaining $10 \%-48.6 \%$ of the variances. However, UE and LE motor functions were not identified as major predictors of variances in these domains. This may be because balance control is more important for mobility and participation in social activities. $\mathrm{Ng}$ illustrated that balance ability was associated with functional mobility in stroke survivors [38]. Patterson et al.'s study asserted that balance function was more important than paretic limb strength for long distance walking in stroke patients with severe gait deficit [39]. In Kollen et al.'s study [40], improvement in standing balance control was more important than improvement in LE strength or synergism in achieving walking ability improvements in patients with stroke. Schmid et al. illustrated that balance function was independently associated with participation in patients with chronic stroke [41]. These findings may suggest balance training should be included in the rehabilitation for enhancing the mobility and participation of HRQOL domains.

Good cognition can predict better memory and communication. We found that MMSE was an important predictor for memory and communication accounting for $22.5 \%-36.3 \%$ of the variances. Our result was similar to Carod-Artal et al.'s [16] study that evaluated the determinants of HRQOL (using SIS 3.0) in 260 patients with stroke and found that MMSE score was a predictor of the memory and communication domains. However, in another article, cognition also affected physical function and other domains of HRQOL. In Patel et al.'s study [42], cognitive impairment (MMSE score <24) was identified as a predictor for lower physical component score and mental component score on SF-36. Jeong et al.'s article [8] also illustrated that MMSE score was independently associated with physical factors, psychological factors, and environmental context of HRQOL. The different results may be due to different predictors and HRQOL measurements among the various studies.

Extremity motor function predicted various HRQOL domains. UE motor function predicted hand function and ADL/IADL of HRQOL, whereas LE motor function was a predictor for the mobility. Nicolas-Larsen et al.'s study [5] found decreased UE motor function was a predictor for poorer composite physical domain (combined strength, ADL/IADL, mobility, and hand function) score on the SIS 3.0 in patients 3-9 months after stroke. Lin et al. [43] found that FMA-UE was a predictor for hand function, $\mathrm{ADL} / \mathrm{IADL}$, and participation/role domains in patients 6 months after stroke. Franceschini et al. [12] found that incomplete LE motor recovery was a predictor for a lower HRQOL in the mobility domain, and incomplete UE motor recovery was a predictor for a lower HRQOL in usual activities and self-care domains. These findings may suggest that treatment strategies should include UE and LE training to promote HRQOL in various domains.

BDI-II predicted HRQOL to a lesser extent than other clinical factors. In our study, BDI-II was a predictor for the memory, emotion and mobility domains, but only accounted for $1.3 \%-6.5 \%$ of the variances. Though the variance is low, depression could affect patient motivation for participating in rehabilitation programs and 
mobility tasks to a certain degree. Froes et al.'s study [13] used BDI to measure the effect of depression on HRQOL (SF-36) and found that BDI was not only a significant factor for mental health, emotional role, social functioning, but also for the vitality scale. Kwok et al. [44] evaluated HRQOL of 268 patients with stroke who had received inpatient rehabilitation for 3 weeks during the acute stage. They followed up these patients at 3, 6, and 12 months after stroke and found that more severe depression was associated with decreased physical, psychological, social interaction, and environmental HRQOL domains [44]. Depression status seems to affect HRQOL of a patient with stroke in both the psychological domain and certain non-psychological domains. These findings may suggest that psychosocial support and treatment of depression may be needed to enhance some HRQOL domains.

Stroke severity predicted the strength domain and the total SIS score in patients with stroke. In this study, NIHSS predicted strength domain, explaining $41.5 \%$ of the variance and predicted total SIS score, explaining $41.7 \%$ of the variance. In Carod-Artal et al.'s article [16], NIHSS $>6$ was identified as a predictor for composite physical domain and multiple domains, including strength, ADL/IADL, participation/role, mobility, and hand function for stroke survivors. Sturm et al's [4] articles showed that the NIHSS score 7 days after stroke could be used to predict total HRQOL score at 2 years after stroke. It seems stroke severity is a robust factor in predicting total HRQOL of patients with stroke, particularly in physical domains.

In our study, stroke side predicted $11.6 \%$ of the variance in the emotion domain, which was greater than was explained by depression. Right hemisphere stroke has a lower HRQOL in the emotion domain than left hemisphere stroke in our study. Rachpukdee et al. [45] also illustrated right hemisphere stroke was a negative predictor for the mental health domain of HRQOL at one month post-stroke. Although right cerebral hemisphere is dominant for sense of emotion [46], some previous articles did not show the same result as ours. In Morris et al. article [10], left hemisphere stroke was significantly associated with, but did not predict poorer emotional reactions. Hopman et al. [22] illustrated the left hemisphere stroke predicted worse emotional functioning than those with right hemisphere stroke at their admission for rehabilitation. However, no difference of stroke side on emotional functioning existed in these patients at their discharge and 6-month follow-up [22]. Although some studies have shown that lesion side had impacts on the emotional domain of HRQOL, other studies have not. One previous study [6] showed that lesion side only had impacts on the body care, movement, and communication, but not on the emotional domain of HRQOL. Another article [9] showed that lesion side did not influence the HRQOL score values in first-ever stroke patients. Therefore, the effect of stroke side on emotion seems to be inconclusive and warrants further study. Lincoln et al. [47] showed that the severity of anxiety and depression markedly increased between 6 months and 5 years after stroke. Therefore, anxiety should also be included in further studies in predicting HRQOL for patients with stroke at both the acute stage and follow-up stages.

The major limitations in this study were participant characteristics and selection of potential predictors. Only participants with unilateral cerebral stroke who were receiving inpatient rehabilitation were included. In addition, other confounding factors influencing HRQOL, such as environmental factors [48, 49], family support [50], psychosocial factors [11,48], or rehabilitation services [51], were not included in this study. For example, ongoing home-based [51] or ambulatory rehabilitation services (physical therapy, occupational therapy, or speech therapy) are probably important confounding factors influencing HRQOL.

\section{Conclusion}

In conclusion, the NIHSS was the major predictor for strength and global HRQOL in patients with stroke. Different factor combinations predicted various HRQOL domains in patients with stroke. BBS, MMSE, FMA-UE, NIHSS scores, and stroke side predicted most HRQOL domains. FIM, FMA-LE, BDI-II scores, duration after onset, and stroke type may influence some HRQOL domains. These findings suggest that these predictors may be useful for the early identification of patients who have the most potential to acquire HRQOL. More importantly, these results may allow clinicians to plan appropriate rehabilitation intervention and enhance HRQOL in patients with stroke by implementing cognitive training (e.g., training involving attention, memory, intellectual execution, visual perception, auditory comprehension, and verbal expression), balance training (e.g., static and dynamic balance training involving sitting balance, standing balance, single leg balance, sit-tostand or stand-to-sit training, and transferring training), and motor functional training (e.g., hand dexterity, object manipulation, visual motor coordination, locomotion, agility, and body coordination). Future studies should focus on longitudinal follow-up and include other potential predictors for HRQOL in patients with stroke.

\section{Abbreviations}

HRQOL: Health-related quality of life; SF-36: Short Form-36; SIS: Stroke Impact Scale; ADL: Activities of daily living; IADL: Instrumental activities of daily living; MMSE: Mini-Mental State Examination; BDI: Beck Depression Inventory; NIHSS: National Institutes of Health Stroke Scale; BBS: Berg Balance Scale; FMA: Fugl-Meyer Assessment; UE: Upper extremity; LE: Lower extremity; FIM: Functional Independence Measure.

\section{Competing interests}

The authors' declare that they have no competing interests. 


\section{Authors' contributions}

C-LC conceived project. C-CT, C-YC, KPHW, and H-CC were responsible for data collection and management. C-LC, C-CT, and C-MC were responsible for data analyses. C-MC wrote the first draft of the article and all authors contributed to the development of the manuscript. All authors read and approved the final manuscript.

\section{Acknowledgments}

We are grateful to the members of the Department of Physical Medicine and Rehabilitation at Chang Gung Memorial Hospital. The Chang Gung Memorial Hospital Research Project Grant financially supported this research under Contract No. CMRPG 391671-3 and CMRPG 3B1141-3.

\section{Author details}

'Department of Physical Medicine and Rehabilitation, Chang Gung Memorial Hospital, Chiayi, No.6, Sec. W., Jiapu Rd., Puzih City, Chiayi County 61363 Taiwan. ${ }^{2}$ School of Medicine, College of Medicine, Chang Gung University, Taoyuan, No.259, Wunhua 1st Rd.Kuei-Shan Dist., Taoyuan City 33302, Taiwan. ${ }^{3}$ Department of Physical Medicine and Rehabilitation, Chang Gung Memoria Hospital, Linkou, No.5, Fushing St. Kuei-Shan Dist., Taoyuan City 33305, Taiwan. ${ }^{4}$ Graduate Institute of Early Intervention, College of Medicine, Chang Gung University, Taoyuan, No.259, Wunhua 1st Rd. Kuei-Shan Dist., Taoyuan City 33302, Taiwan. ${ }^{5}$ Department of Industrial Engineering \& Management, National Taipei University of Technology, No.1, Sec. 3, Zhongxiao E. Rd., Daan Dist., Taipei City 10608, Taiwan.

\section{Received: 18 March 2015 Accepted: 24 July 2015 Published online: 05 August 2015}

\section{References}

1. Lai SM, Perera S, Duncan PW, Bode R. Physical and social functioning after stroke: comparison of the Stroke Impact Scale and Short Form-36. Stroke. 2003;34:488-93.

2. Coons SJ, Rao S, Keininger DL, Hays RD. A comparative review of generic quality-of-life instruments. Pharmacoeconomics. 2000;17:13-35.

3. Xie J, Wu EQ, Zheng ZJ, Croft JB, Greenlund KJ, Mensah GA, et al. Impact of stroke on health-related quality of life in the noninstitutionalized population in the United States. Stroke. 2006;37:2567-72.

4. Sturm JW, Donnan GA, Dewey HM, Macdonell RA, Gilligan AK, Srikanth V, et al. Quality of life after stroke: the North East Melbourne Stroke Incidence Study (NEMESIS). Stroke. 2004;35:2340-5.

5. Nichols-Larsen DS, Clark PC, Zeringue A, Greenspan A, Blanton S. Factors influencing stroke survivors' quality of life during subacute recovery. Stroke. 2005;36:1480-4.

6. de Haan RJ, Limburg M, Van der Meulen JH, Jacobs HM, Aaronson NK. Quality of life after stroke. Impact of stroke type and lesion location. Stroke. 1995;26:402-8

7. Shinohara Y. Factors affecting health-related quality of life assessed with the SF-36v2 health survey in outpatients with chronic-stage ischemic stroke in Japan-cross-sectional analysis of the OASIS study. Cerebrovasc Dis. 2010;29:361-71.

8. Jeong BO, Kang HJ, Bae KY, Kim SW, Kim JM, Shin IS, et al. Determinants of quality of life in the acute stage following stroke. Psychiatry Investig. 2012;9:127-33

9. Gurcay E, Bal A, Cakci A. Health-related quality of life in first-ever stroke patients. Ann Saudi Med. 2009;29:36-40.

10. Morris JH, van Wijck F, Joice S, Donaghy M. Predicting health related quality of life 6 months after stroke: the role of anxiety and upper limb dysfunction. Disabil Rehabil. 2013;35:291-9.

11. Alguren B, Fridlund B, Cieza A, Sunnerhagen KS, Christensson L. Factors associated with health-related quality of life after stroke: a 1-year prospective cohort study. Neurorehabil Neural Repair. 2012;26:266-74.

12. Franceschini M, La Porta F, Agosti M, Massucci M. Is health-related-quality of life of stroke patients influenced by neurological impairments at one year after stroke? Eur J Phys Rehabil Med. 2010;46:389-99.

13. Froes KS, Valdes MT, Lopes Dde P, Silva CE. Factors associated with healthrelated quality of life for adults with stroke sequelae. Arq Neuropsiquiatr. 2011;69:371-6.

14. Fatoye FO, Komolafe MA, Eegunranti BA, Adewuya AO, Mosaku SK, Fatoye GK. Cognitive impairment and quality of life among stroke survivors in Nigeria. Psychol Rep. 2007;100:876-82
15. Carod-Artal FJ, Coral LF, Trizotto DS, Moreira CM. The stroke impact scale 3.0: evaluation of acceptability, reliability, and validity of the Brazilian version. Stroke. 2008;39:2477-84.

16. Carod-Artal FJ, Trizotto DS, Coral LF, Moreira CM. Determinants of quality of life in Brazilian stroke survivors. J Neurol Sci. 2009;284:63-8.

17. Andrews K, Brocklehurst JC, Richards B, Laycock PJ. The rate of recovery from stroke - and its measurement. Int Rehabil Med. 1981;3:155-61.

18. Mackenzie AE, Chang AM. Predictors of quality of life following stroke. Disabil Rehabil. 2002;24:259-65.

19. Sprigg N, Gray LJ, Bath PM, Christensen H, De Deyn PP, Leys D, et al. Quality of life after ischemic stroke varies in western countries: data from the tinzaparin in Acute Ischaemic Stroke Trial (TAIST). J Stroke Cerebrovasc Dis. 2012;21:587-93

20. Owolabi MO. Consistent determinants of post-stroke health-related quality of life across diverse cultures: Berlin-Ibadan study. Acta Neurol Scand. 2013;128:311-20

21. Duncan PW, Horner RD, Reker DM, Samsa GP, Hoenig H, Hamilton B, et al. Adherence to postacute rehabilitation guidelines is associated with functional recovery in stroke. Stroke. 2002;33:167-77.

22. Hopman WM, Verner J. Quality of life during and after inpatient stroke rehabilitation. Stroke. 2003:34:801-5.

23. Kim S-K, Kim S-H, Jo M-W, Lee S-i. Estimation of minimally important differences in the EQ-5D and SF-6D indices and their utility in stroke. Health Qual Life Outcomes. 2015;13:32.

24. Ghatnekar O, Eriksson M, Glader EL. Mapping health outcome measures from a stroke registry to EQ-5D weights. Health Qual Life Outcomes. 2013;11:34.

25. Kim SH, Kim SO, Lee SI, Jo MW. Deriving a mapping algorithm for converting SF-36 scores to EQ-5D utility score in a Korean population. Health Qual Life Outcomes. 2014;12:145.

26. Lin KC, Fu T, Wu CY, Hsieh CJ. Assessing the stroke-specific quality of life for outcome measurement in stroke rehabilitation: minimal detectable change and clinically important difference. Health Qual Life Outcomes. 2011;9:5.

27. Hobart JC, Williams LS, Moran K, Thompson AJ. Quality of life measurement after stroke: uses and abuses of the SF-36. Stroke. 2002;33:1348-56.

28. Salter $\mathrm{KL}$, Moses MB, Foley NC, Teasell RW. Health-related quality of life after stroke: what are we measuring? Int J Rehabil Res. 2008;31:111-7.

29. Wu CY, Yang CL, Chuang LL, Lin KC, Chen HC, Chen MD, et al. Effect of therapist-based versus robot-assisted bilateral arm training on motor control, functional performance, and quality of life after chronic stroke: a clinical trial. Phys Ther. 2012;92:1006-16.

30. Lin KC, Fu T, Wu CY, Wang YH, Liu JS, Hsieh CJ, et al. Minimal detectable change and clinically important difference of the Stroke Impact Scale in stroke patients. Neurorehabil Neural Repair. 2010;24:486-92.

31. Duncan PW, Bode RK, Min Lai S, Perera S. Rasch analysis of a new stroke-specific outcome scale: the Stroke Impact Scale. Arch Phys Med Rehabil. 2003:84:950-63.

32. Cockrell JR, Folstein MF. Mini-Mental State Examination (MMSE). Psychopharmacol Bull. 1988;24:689-92.

33. Steer RA, Ball R, Ranieri WF, Beck AT. Dimensions of the Beck Depression Inventory-II in clinically depressed outpatients. J Clin Psychol. 1999;55:117-28.

34. Brott T, Adams Jr HP, Olinger CP, Marler JR, Barsan WG, Biller J, et al. Measurements of acute cerebral infarction: a clinical examination scale. Stroke. 1989;20:864-70

35. Berg KO, Maki BE, Williams Jl, Holliday PJ, Wood-Dauphinee SL. Clinical and laboratory measures of postural balance in an elderly population. Arch Phys Med Rehabil. 1992;73:1073-80.

36. Fugl-Meyer AR, Jaasko L, Leyman I, Olsson S, Steglind S. The post-stroke hemiplegic patient. 1. a method for evaluation of physical performance. Scand J Rehabil Med. 1975;7:13-31.

37. Ottenbacher KJ, Hsu Y, Granger CV, Fiedler RC. The reliability of the functional independence measure: a quantitative review. Arch Phys Med Rehabil. 1996;77:1226-32

38. Ng SS. Contribution of subjective balance confidence on functional mobility in subjects with chronic stroke. Disabil Rehabil. 2011;33:2291-8.

39. Patterson SL, Forrester LW, Rodgers MM, Ryan AS, Ivey FM, Sorkin JD, et al. Determinants of walking function after stroke: differences by deficit severity. Arch Phys Med Rehabil. 2007;88:115-9.

40. Kollen B, van de Port I, Lindeman E, Twisk J, Kwakkel G. Predicting improvement in gait after stroke: a longitudinal prospective study. Stroke. 2005;36:2676-80. 
41. Schmid AA, Van Puymbroeck M, Altenburger PA, Dierks TA, Miller KK, Damush TM, et al. Balance and balance self-efficacy are associated with activity and participation after stroke: a cross-sectional study in people with chronic stroke. Arch Phys Med Rehabil. 2012:93:1101-7.

42. Patel MD, McKevitt C, Lawrence E, Rudd AG, Wolfe CD. Clinical determinants of long-term quality of life after stroke. Age Ageing. 2007;36:316-22.

43. Lin KC, Fu T, Wu CY, Hsieh YW, Chen CL, Lee PC. Psychometric comparisons of the Stroke Impact Scale 3.0 and Stroke-Specific Quality of Life Scale. Qual Life Res. 2010;19:435-43.

44. Kwok T, Lo RS, Wong E, Wai-Kwong T, Mok V, Kai-Sing W. Quality of life of stroke survivors: a 1-year follow-up study. Arch Phys Med Rehabil. 2006;87:1177-82.

45. Rachpukdee S, Howteerakul N, Suwannapong N, Tang-Aroonsin S. Quality of life of stroke survivors: a 3-month follow-up study. J Stroke Cerebrovasc Dis. 2013;22:e70-8.

46. Devinsky O. Right Cerebral Hemisphere Dominance for a Sense of Corporeal and Emotional Self. Epilepsy \& Behavior. 2000;1:60-73.

47. Lincoln NB, Brinkmann N, Cunningham S, Dejaeger E, De Weerdt W, Jenni W, et al. Anxiety and depression after stroke: a 5 year follow-up. Disabil Rehabil. 2013;35:140-5

48. Manimmanakorn N, Vichiansiri R, Nuntharuksa C, Permsirivanich W, Kuptniratsaikul V. Quality of life after stroke rehabilitation among urban vs. rural patients in Thailand. J Med Assoc Thai. 2008;91:394-9.

49. Kwok T, Pan JH, Lo R, Song X. The influence of participation on health-related quality of life in stroke patients. Disabil Rehabil. 2011;33:1990-6.

50. Carod-Artal J, Egido JA, Gonzalez JL. Varela de Seijas E. Quality of life among stroke survivors evaluated 1 year after stroke: experience of a stroke unit. Stroke. 2000;31:2995-3000.

51. Studenski S, Duncan PW, Perera S, Reker D, Lai SM, Richards L. Daily functioning and quality of life in a randomized controlled trial of therapeutic exercise for subacute stroke survivors. Stroke. 2005;36:1764-70.

\section{Submit your next manuscript to BioMed Central and take full advantage of:}

- Convenient online submission

- Thorough peer review

- No space constraints or color figure charges

- Immediate publication on acceptance

- Inclusion in PubMed, CAS, Scopus and Google Scholar

- Research which is freely available for redistribution 